\title{
Arthroscopic Treatment of a Type II Superior Labrum Anterior to Posterior (SLAP) Lesion Combined with a Bankart Lesion: Comparative Study between Debridement and Repair of Type II SLAP Lesion by the Status of Lesion
}

\author{
Sung Hyun Lee*, Min Su Joo*, Kyeong Hoon Lim, Jeong Woo Kim \\ Department of Orthopedic Surgery, Wonkwang University Hospital, Iksan, Korea
}

\begin{abstract}
Background: The purpose of this study is to evaluate results of superior labrum anterior to posterior (SLAP) repairs and debridement of type II SLAP lesions combined with Bankart lesions.

Methods: Between 2010 and 2014, total 58 patients with anterior shoulder instability due to a Bankart lesion combined with a type II SLAP lesion were enrolled. Patients were divided into two groups: group C consisted of 30 patients, each with a communicated Bankart and type II SLAP lesion and group NC consisted of 28 patients, each with a non-communicated Bankart and type II SLAP lesion. Bankart repairs were performed for all patients. SLAP lesions were repaired in group C and debrided in group NC. Clinical results were analysed to compare groups $\mathrm{C}$ and NC by using the visual analogue scale pain score, American Shoulder and Elbow Surgeons score, Constant scores, Rowe score for instability and range of motion assessments.

Results: The clinical scores were improved in both groups at final follow-up. Also, there were no differences between two groups. No significant difference was found in terms of the range of motion measured at the last follow-up. The number of suture anchors used was significantly higher in group C than in group NC (5.6 vs. 3.8; $p=0.021)$.

Conclusions: In this study, it is considered that Bankart repair and SLAP debridement could be a treatment option in patients with a non-communicated type II SLAP lesion combined with a Bankart lesion (study design: IV, therapeutic study, case series).
\end{abstract}

(Clin Shoulder Elbow 2018;21(1):37-41)

Key Words: Bankart lesion; Glenoid labrum; Superior labrum from anterior to posterior; Debridement

\section{Introduction}

Type II superior labrum anterior to posterior (SLAP) lesions can occur as isolated injuries, but may also be accompanied by Bankart lesions (tears of the anteroinferior labrum) in cases of recurrent dislocation, classified by Maffet et al. ${ }^{1)}$ as type V SLAP lesions. Combined lesions of the glenoid labrum are not uncommon, especially in chronic cases, as the severity of labrum lesions increases with time and number of dislocations. ${ }^{2}$ Much of the literature reports that a combination of a Bankart and a type
II SLAP lesion occurred in approximately $20 \%$ of cases with anterior instability. ${ }^{3,4)}$ Takase ${ }^{5)}$ suggested that there were two types of combined SLAP and Bankart lesions. One is a SLAP lesion communicated to a Bankart lesion, and the other exists with no communication between the Bankart lesion and SLAP lesion (Fig. 1). It is known that type II SLAP lesions result in increased glenohumeral translation and-when combined with a Bankart lesion-increase shoulder instability. ${ }^{6,7}$

The Bankart lesion has been described as the 'essential lesion' in patients with anterior shoulder instability. ${ }^{8,9)}$ Repair of a

Received November 15, 2017. Revised January 21, 2018. Accepted February 12, 2018.

*These authors contributed equally to this article as co-first authors.

Correspondence to: Jeong Woo Kim

Department of Orthopedic Surgery, Wonkwang University Hospital, 895 Muwang-ro, Iksan 54538, Korea

Tel: +82-63-859-1360, Fax: +82-63-852-9329, E-mail: serina@wonkwang.ac.kr

IRB approval: Wonkwang University Hospital (No. WKUHIRB 201507-HRE-065).

Financial support: This study was supported by an Wonkwnag University research grant. Conflict of interests: None. 
A

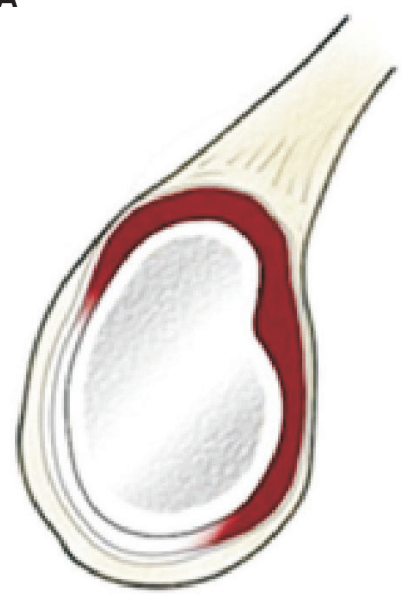

B

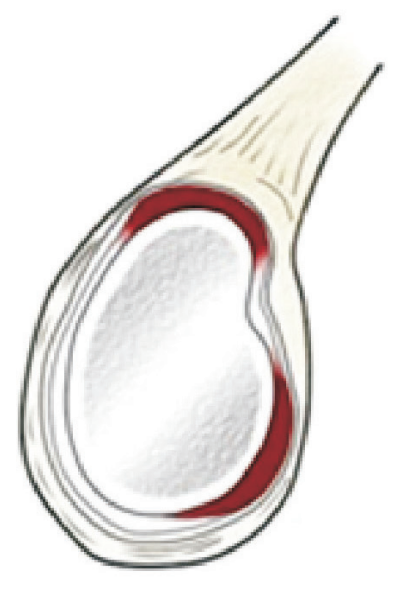

Fig. 1. Schematic diagram of (A) communicating Bankart and superior labrum anterior to posterior (SLAP) lesions and (B) non-communicating Bankart and SLAP lesions.

Bankart lesion is the standard choice in the treatment of anterior shoulder instability. ${ }^{10)}$ Conversely, controversy exists over proper repair techniques of SLAP lesions. Debridement alone resulted in satisfying results in some studies, ${ }^{11,12)}$ while it produced poor results and a high potential for instability in others. ${ }^{13,14)}$ When a combined lesion of SLAP and Bankart was present, the treatment of the SLAP lesion after surgical repair of the Bankart lesion has not been clearly defined. There is limited literature about these combined glenoid lesions.

Therefore, authors hypothesize that a type II SLAP lesion develops secondarily when chronic instability caused by repeated dislocation or subluxation episodes in traumatic Bankart lesion is present, possibly causing a non-communicated type II SLAP lesion. In these cases, authors consider that repair of the SLAP lesion is not necessary, as it is not an essential lesion causing shoulder instability, rather, a secondary lesion. Therefore, repairs of the Bankart lesions were performed and only a debridement was performed for the type II SLAP lesions. However, if type II SLAP lesions were communicated with Bankart lesions, the lesions are thought to be caused by a strong force at the initial trauma, occurring at the same time as the Bankart lesion. In these communicated lesions, authors hypothesize that SLAP lesions and Bankart lesions - thought to be essential lesions of anterior shoulder instability - were all repaired. The purpose of this retrospective study is to compare the clinical outcomes of Bankart lesions alone being repaired (SLAP lesion debridement) in patients with Bankart lesions combined with non-communicated SLAP lesions and all lesions being repaired in patients with Bankart lesions combined with communicated SLAP lesions.

\section{Methods}

\section{Patients Selection}

This study targeted patients who had undergone arthroscopic treatment due to anterior shoulder instability, which was performed by a single surgeon in our institution. Inclusion criteria are as follows: (i) An anterior shoulder instability test (more than 1 episode of traumatic shoulder dislocation) with positive anterior apprehension, (ii) the combination of a Bankart lesion and a type II SLAP lesion, (iii) a follow-up performed minimally 24 months postoperatively, and (iv) a normal contralateral shoulder. Exclusion criteria were as follows: (i) Patients with a bony Bankart lesion or an anterior glenoid defect of more than 25\%, (ii) generalized ligamentous laxity, (iii) humeral avulsion of the glenohumeral ligament (HAGL). Patients were divided into two groups according to the presence of and communication between Bankart and type II SLAP lesions.

\section{Demographics and Clinical Assessment}

Age, gender, mean number of dislocations, follow-up period, side of the affecting shoulder, time from injury to surgery, and sports activity level were checked preoperatively to exclude the interference factors between two groups. Clinical data were evaluated preoperatively and at final follow-up. The visual analogue scale (VAS) pain score, American Shoulder and Elbow Surgeons (ASES) score, Constant score, Rowe score, and passive shoulder range of motion (ROM) were used for clinical outcome assessment. Improvement of the anterior apprehension test and recurrence of shoulder dislocation were also checked postoperatively.

\section{Surgical Technique}

Surgical treatments were performed for patients who had anterior instability-identified by the anterior apprehension test or relocation test-and difficulty in daily activity and persistent pain. The patient was positioned in the lateral decubitus position and before surgery, an examination under anesthesia for anterior translation with the arm in abduction was performed and instability classified according to Altchek and Dines. ${ }^{15)}$ Standard arthroscopic portals - including both posterior and anteriorwere used in most cases. Examination of joint and labral condition was performed using the posterior portals as viewing portals. The inferior glenoid measurement was performed using a probe. Usually, three to four anchors for repair of a Bankart lesion were used. For non-communicated SLAP lesions, only a debridement was done. For communicated SLAP lesions, one or two anchors were used (after completion of Bankart repair). We omitted closure of the anterosuperior labrum during SLAP lesion repair to reduce the risk of postoperative stiffness.

\section{Postoperative Management}

Postoperatively, the arms of the patients were kept in a shoul- 
der sling with an abduction pillow at neutral rotation and $20^{\circ}$ abduction. Three weeks after the surgery, progressive self-assisted shoulder elevation and external rotation were initiated. Postoperatively, active ROM exercises were permitted at 6 weeks, rotator cuff strengthening at 12 weeks, and full participation in sports at 6 months.

\section{Statistical Analysis}

Statistical analysis was conducted using IBM SPSS for Windows Release ver. 23.0 (IBM Co., Armonk, NY, USA) with a 95\% confidence level. A student t-test and Mann-Whitney test were used for comparison between the two groups and The Wilcoxon signed rank test was used for comparisons of preoperative and postoperative conditions. The level of significance was $p$ value $<0.05$.

\section{Ethics Approval and Consent to Participate}

The protocol of this retrospective comparative study was approved by Wonkwang University Hospital Institutional Review Board (approval number: WKUHIRB 201507-HRE-065). Informed consents were obtained from all participants.

\section{Results}

A retrospective cohort of 91 arthroscopic surgeries for anterior shoulder instability performed between from March 2005 and February 2014 was used. Of the 91 cases, 33 were excluded according to the exclusion criteria: (i) bony Bankart lesions or anterior glenoid defects of more than $25 \%(n=7)$; (ii) generalized ligamentous laxity ( $\mathrm{n}=15)$; (iii) HAGL lesion ( $\mathrm{n}=2)$; and (iv) no follow-up before 24 months $(n=9)$. Consequently, 58 patients with a median age of 25.1 years (range, 18-43 years) and mean follow-up period of 35.1 months (range, 24-122 months) met the inclusion criteria and were sorted into two groups. Group C consisted of 30 patients with communicated Bankart and type II SLAP lesions and group NC consisted of 28 patients with noncommunicated Bankart and type II SLAP lesion.

\section{Demographics}

In the demographic patient data, patients in group NC had more dislocations (5.1 times vs. 2.3 times; $p<0.01$ ) preoperatively and time from injury to surgery (5.6 years vs. 2.5 years; $p<0.01$ ) compared with group C (Table 1).

Table 1. Demographic Data of Patients

\begin{tabular}{|c|c|c|c|}
\hline Variable & Group C & Group NC & $p$-value \\
\hline No. of patient & 30 & 28 & NS \\
\hline Median age (yr) & $22(18-41)$ & $27(19-43)$ & NS \\
\hline Follow-up time (mo) & $36(24-122)$ & $34(24-112)$ & NS \\
\hline Gender (male/female) & $28(93.3) / 2(6.7)$ & $25(89.3) / 3(10.7)$ & NS \\
\hline Dominant arm/non-dominant arm & $19(63.3) / 11(36.7)$ & $19(67.9) / 9(32.1)$ & NS \\
\hline Mean no. of dislocation & $2.3 \pm 1.4$ & $5.1 \pm 3.1$ & $<0.01$ \\
\hline$(1 /$ more than 1$)$ & $5(16.7) / 25(83.3)$ & $2(3.6) / 26(96.4)$ & NS \\
\hline TFI (yr) & $2.5(0.3-10.0)$ & $5.6(0.5-20.0)$ & $<0.01$ \\
\hline Sport activity level & & & NS \\
\hline High & $10(33.3)$ & $8(28.6)$ & \\
\hline Moderate & $17(56.7)$ & $16(57.1)$ & \\
\hline Low & $3(10.0)$ & $4(14.3)$ & \\
\hline \multicolumn{4}{|l|}{ Preoperative score } \\
\hline VAS & $5.0(3-8)$ & $5.3(4-8)$ & NS \\
\hline ASES & $55.2(42-64)$ & $51.5(40-62)$ & NS \\
\hline Constant & $50.5(34-57)$ & $49.1(36-60)$ & NS \\
\hline Rowe & $34.6(18-46)$ & $29.5(15-42)$ & NS \\
\hline \multicolumn{4}{|l|}{ Preoperative ROM $\left(^{\circ}\right)$} \\
\hline Forward elevation & $173.5(160-180)$ & $167.4(150-180)$ & NS \\
\hline External rotation & $74.6(50-90)$ & $73.2(60-90)$ & NS \\
\hline
\end{tabular}

Values are presented as number only, median (range), number (\%), or mean \pm standard deviation.

Group C: with a communicated Bankart and type II superior labrum anterior to posterior (SLAP) lesion, Group NC: with a non-communicated Bankart and type II SLAP lesion, TFI: time from injury to surgery, VAS: visual analogue scale, ASES: American Shoulder and Elbow Surgeons, ROM: range of motion, NS: not significant. 
Other demographic data were well-matched and not statistically different (Table 1). ROM was nearly intact, and apprehension test results were positive in all patients preoperatively.

\section{Clinical Results}

The postoperative ASES, Constant, Rowe, and VAS scores are presented in Table 2. These clinical outcomes showed no significant difference between the two groups. All shoulder scores improved postoperatively in both groups $(p<0.05)$. Between the two groups, no significant difference was found regarding the ROM (forward elevation and external rotation) measured at the last follow-up (Table 2). One patient from group $\mathrm{C}$ redislocated his shoulder while competitively exercising, and one patient from group NC reported an episode of subluxation when his shoulder was placed by force in abduction and external rotation. Two patients from group $\mathrm{C}$ and three patients from group $\mathrm{NC}$ had a positive apprehension test result at the last follow-up evaluation. There were no significant differences regarding the number of complications between the two groups.

\section{Discussion}

The most important findings of this study were that the clinical outcomes of the Bankart lesion only repairs (SLAP lesion debridement) in patients with Bankart lesions combined noncommunicated SLAP lesions was comparable to all lesions being repaired in patients with Bankart lesions combined with a communicated SLAP lesion.

Regarding the injury mechanism of SLAP lesions, Snyder et

Table 2. Clinical Results at the Last Follow-up

\begin{tabular}{lcll}
\hline \multicolumn{1}{c}{ Variable } & Group C & Group NC & $p$-value \\
\hline Postoperative score & & & \\
\hline VAS & $0.89(0-3)$ & $0.84(0-4)$ & NS \\
\hline ASES & $94.7(52-100)$ & $95.2(54-100)$ & NS \\
\hline Constant & $91.6(54-100)$ & $92.5(60-100)$ & NS \\
\hline Rowe & $90.5(52-100)$ & $89.2(58-100)$ & NS \\
Postoperative ROM & & & \\
\hline Forward elevation & $170.5(160-180)$ & $169.3(150-180)$ & NS \\
\hline External rotation & $71.6(50-90)$ & $71.2(60-90)$ & NS \\
\hline Return to work & $30(100)$ & $28(100)$ & NS \\
Return to sports & $27(90.0)$ & $26(92.9)$ & NS \\
\hline Recurrences & $1(3.3)$ & $1(3.6)$ & NS \\
\hline Positive apprehension test & $2(6.7)$ & $3(10.7)$ & NS \\
\hline
\end{tabular}

Values are presented as median (range) or number (\%).

Group C: with a communicated Bankart and type II superior labrum anterior to posterior (SLAP) lesion, Group NC: with a non-communicated Bankart and type II SLAP lesion, VAS: visual analogue scale, ASES: American Shoulder and Elbow Surgeons, ROM: range of motion, NS: not significant. al. ${ }^{16)}$ reported that the force of pushing up the glenoid by the humeral head upon touching the ground with the hand while in abduction of the shoulder joint caused type I or II SLAP lesions, and the addition of external rotation to this axially compressed condition led to type III or IV lesions complicated by anterior instability. In some cases, recurrent dislocation or subluxation and traction by the tendon of the long head of the biceps brachii may lead to a SLAP lesion concomitant with a Bankart lesion. ${ }^{5)}$ This is an extensive lesion that involves the biceps anchor leading to a poor outcome when treated conservatively, such as external rotation. Kim et al. ${ }^{17)}$ suggested that the possibility of spontaneous healing of SLAP lesions over time is less likely in type $\mathrm{V}$ lesions than in the recurrent group. Takase $e^{5)}$ primarily described the presence of communication between Bankart and SLAP lesions. He suggested that when SLAP lesions communicated to Bankart lesions, an enlargement on the detachment of the anterior labrum from the anterior margin of the glenoid - which occurred from repeated dislocation or subluxation - may have led to the SLAP lesions. In those cases, the repair of only the Bankart lesion was enough. ${ }^{5)}$ Existing literature states that for SLAP lesions, only debridement or reattachment to the superior glenoid need to be performed, considering whether these lesions communicated to Bankart lesions. ${ }^{5}$ However, the hypothesis of this study was contrary to the opinion suggested by Takase. ${ }^{5)}$ Authors of this study considered that SLAP lesions non-communicated to Bankart lesions were occurred by microtrauma from repeated dislocations or subluxations; in those cases, only the Bankart lesions were repaired and SLAP lesions were debrided. The current study also considered that SLAP lesions communicated to Bankart lesions were occurred from high energy trauma at initial injury, and it acted as the primary lesion of anterior shoulder instability; in these cases, SLAP lesions and Bankart lesions were all repaired. Yiannakopoulos et al. ${ }^{4)}$ reported on intra-articular lesions with acute and chronic traumatic anterior shoulder instability. It was concluded from that study that secondary intraarticular lesions are more frequent in patients with chronic cases, probably as a result of repeated dislocation or subluxation episodes. These findings were in accordance with the hypothesis of this study that reported a high recurrence rate of dislocation and longer time from injury to surgery in the NC group. It is assumed that the longer interval between injury and surgery and number of dislocations played a significant role in the development of secondary SLAP lesions of shoulder instability.

Repairs of relatively extensive labral tears-such as combined Bankart and SLAP lesions - can result in restrictions on the ROM. According to Warner et al., ${ }^{18)}$ external rotation was restricted by $6^{\circ}$ compared to the unaffected side when the shoulder was at $90^{\circ}$ abduction. Cho et al. ${ }^{19)}$ reported favourable clinical results in patients with recurrent shoulder dislocations and type $\mathrm{V}$ tears compared with those with an isolated Bankart lesion. There were no limitations in ROM of more than $10^{\circ}$ at 
last follow-up, although patients with type $\mathrm{V}$ tears had a slower postoperative recovery of shoulder motion. Also in this study, no limitations were present in ranges of motion of more than $10^{\circ}$ at last follow-up compared to the contralateral shoulder in both groups. The reason of this result was might be due to omit closure of anterosuperior labrum. In fact, it has been reported that limited joint mobility is a relatively common complication in patients with SLAP lesion repairs. However, Oh et al. ${ }^{20)}$ reduced the risk of postoperative stiffness by omitting closure of the anterosuperior labrum during SLAP lesion repair to reduce tension in the rotator interval. Therefore, it was postulated that the recovery of the ROM was caused by omitting closure of the anterosuperior labrum in patients at our institution.

Some limitations of this study include this being is a retrospective study, meaning data was collected prospectively. Secondly, this study included a small study population and relatively short follow-up period. Therefore, this study indicates that studies involving more cases and long-term follow-up periods should be performed in order to clarify the reason for the delay in the recovery of the ranges of motion. Additionally, two groups were not affected by the same condition. Specifically, repairs of all were done for the communicated SLAP lesion groups, and only Bankart repairs were done for non-communicated SLAP lesion group.

\section{Conclusion}

In the current study, authors have obtained satisfactory results in both groups. Also, although the non-communicated type II SLAP lesion accompanying the Bankart lesion was not repaired, it would not affect shoulder stability or the clinical result. Unnecessary repairs for the SLAP lesion non-communicated to Bankart lesions should, therefore, be avoided.

\section{References}

1. Maffet MW, Gartsman GM, Moseley B. Superior labrumbiceps tendon complex lesions of the shoulder. Am J Sports Med. 1995;23(1):93-8.

2. Habermeyer P, Gleyze P, Rickert M. Evolution of lesions of the labrum-ligament complex in posttraumatic anterior shoulder instability: a prospective study. J Shoulder Elbow Surg. 1999; 8(1):66-74.

3. Garofalo R, Mocci A, Moretti B, et al. Arthroscopic treatment of anterior shoulder instability using knotless suture anchors. Arthroscopy. 2005;21(11):1283-9.

4. Yiannakopoulos CK, Mataragas E, Antonogiannakis E. A comparison of the spectrum of intra-articular lesions in acute and chronic anterior shoulder instability. Arthroscopy. 2007;23(9):985-90.

5. Takase K. Risk of motion loss with combined Bankart and SLAP repairs. Orthopedics. 2009;32(8). doi: 10.3928/0147744720090624-05.

6. McMahon PJ, Burkart A, Musahl V, Debski RE. Glenohumeral translations are increased after a type II superior labrum anterior-posterior lesion: a cadaveric study of severity of passive stabilizer injury. J Shoulder Elbow Surg. 2004;13(1):39-44.

7. Mihata T, McGarry MH, Tibone JE, Fitzpatrick MJ, Kinoshita M, Lee TQ. Biomechanical assessment of Type II superior labral anterior-posterior (SLAP) lesions associated with anterior shoulder capsular laxity as seen in throwers: a cadaveric study. Am J Sports Med. 2008;36(8):1604-10.

8. Bankart AS. The pathology and treatment of recurrent dislocation of the shoulder-joint. Br J Surg. 1938;26:23-9.

9. Lenters TR, Franta AK, Wolf FM, Leopold SS, Matsen FA 3rd. Arthroscopic compared with open repairs for recurrent anterior shoulder instability. A systematic review and meta-analysis of the literature. J Bone Joint Surg Am. 2007;89(2):244-54.

10. Mohtadi NG, Bitar IJ, Sasyniuk TM, Hollinshead RM, Harper WP. Arthroscopic versus open repair for traumatic anterior shoulder instability: a meta-analysis. Arthroscopy. 2005;21(6):652-8.

11. Depuy J, Steiner ME. Isolated shoulder labral tears: treatment and correlation with labral anatomy. Orthop Trans. 1990; $14(2): 244$.

12. Resch H, Golser K, Thoeni H, Sperner G. Arthroscopic repair of superior glenoid labral detachment (the SLAP lesion). J Shoulder Elbow Surg. 1993;2(3):147-55.

13. Ogilvie-Harris DJ, Wiley AM. Arthroscopic surgery of the shoulder. A general appraisal. J Bone Joint Surg Br. 1986;68(2):201-7.

14. Pappas AM, Goss TP, Kleinman PK. Symptomatic shoulder instability due to lesions of the glenoid labrum. Am J Sports Med. 1983;11(5):279-88.

15. Altchek DW, Dines DM. Shoulder injuries in the throwing athlete. J Am Acad Orthop Surg. 1995;3(3):159-65.

16. Snyder SJ, Karzel RP, Del Pizzo W, Ferkel RD, Friedman MJ. SLAP lesions of the shoulder. Arthroscopy. 1990;6(4):274-9.

17. Kim DS, Yi CH, Yoon YS. Arthroscopic repair for combined Bankart and superior labral anterior posterior lesions: a comparative study between primary and recurrent anterior dislocation in the shoulder. Int Orthop. 2011;35(8):1187-95.

18. Warner JJ, Kann S, Marks P. Arthroscopic repair of combined Bankart and superior labral detachment anterior and posterior lesions: technique and preliminary results. Arthroscopy. 1994;10(4):383-91.

19. Cho HL, Lee CK, Hwang TH, Suh KT, Park JW. Arthroscopic repair of combined Bankart and SLAP lesions: operative techniques and clinical results. Clin Orthop Surg. 2010;2(1):39-46.

20. Oh JH, Kim SH, Lee HK, Jo KH, Bae KJ. Trans-rotator cuff portal is safe for arthroscopic superior labral anterior and posterior lesion repair: clinical and radiological analysis of 58 SLAP lesions. Am J Sports Med. 2008;36(10):1913-21. 